\title{
Consensus of second-order nonlinear multi-agent systems via sliding mode observer and controller
}

\author{
Xiaolei $\mathrm{Li}^{1}$, Xiaoyuan $\mathrm{Luo}^{1,2, *}$, Shaobao $\mathrm{Li}^{1}$, Jianjin $\mathrm{Li}^{1}$, and Xinping Guan ${ }^{3}$ \\ 1. School of Electrical Engineering, Yanshan University, Qinhuangdao 066004, China; \\ 2. Tandon School of Engineering, New York University, New York 11201, USA; \\ 3. Institute of Electronic, Information and Electrical Engineering, Shanghai Jiao Tong University, Shanghai 200240, China
}

\begin{abstract}
This paper investigates the consensus problem of second-order nonlinear multi-agent systems (MASs) via the sliding mode control (SMC) approach. The velocity of each agent is assumed to be unmeasurable. A second-order sliding mode observer is designed to estimate the velocity. Then a distributed discontinuous control law based on first-order SMC is presented to solve the consensus problem. Moreover, to overcome the chatting problem, two controllers based on the boundary layer method and the super-twisting algorithm respectively are presented. It is shown that the MASs will achieve consensus under some given conditions. Some examples are provided to demonstrate the effectiveness of the proposed control laws.
\end{abstract}

Keywords: nonlinear multi-agent system, sliding mode observer, consensus, sliding mode controller.

DOI: $10.21629 /$ JSEE.2017.04.15

\section{Introduction}

Distributed cooperative control of multi-agent systems (MASs), such as formation [1,2], flocking [3,4], synchronization of coupled oscillators $[5,6]$, has drawn much attention as a result of their scalability and robustness in node failures. As an important fundamental problem of above, consensus with its aim at making the agents swarm achieve an identical state has been extensively studied.

Recently, many results have been made on consensus control for both first-order and second-order MASs [79]. In [7], Yu et al. investigated second-order consensus in MASs with sampling both current and some past position data. Zhu et al. considered the robust second-order consensus tracking problem for the MASs with disturbances and unmodeled agent dynamics [8]. Lin and Jia investigated the second-order consensus problem of discrete-time

Manuscript received September 23, 2015.

*Corresponding author.

This work was supported by the National Natural Science Foundation of China (61375105; 61403334).
MASs with nonuniform time-delays and switching topologies [9]. Furthermore, some other control strategies have been developed for consensus of MASs, for instance $H_{\infty}$ control [10,11], sampled-data control [12], pinning control $[13,14]$, sliding mode control (SMC) $[15,16]$ and robust control $[8,17-19]$. Some constraints including time delay [12], data packet dropout [12] and parameter uncertainties $[11,17]$ have also been considered. Those aforementioned studies are mostly considered the consensus problem of linear MASs. However, there are still many challenges for the nonlinear MASs consensus control problem, especially with unmeasurable states.

Unmeasurable states are usually observed by designing linear observers in existing researches. For example, in [20], a novel consensus protocol based on the state observer with a fast convergence speed was presented for linear MASs, where the unmeasurable states are observed by the well designed linear observers. In [21], Hu et al. considered the distributed pinning consensus control problem for the nonlinear MASs with the local line observer and distributed pinning observer. It is noted that, the linear approaches cannot guarantee the observer and controller converge in finite time for the nonlinear system with uncertainties.

SMC has been widely used to design observers and consensus protocols for MASs [22-24] as a result of its advantages, for instance, high reliability, strong robustness, fast system response and so on. In [22], the SMC is applied for first-order linear MASs with disturbances. In [23-26], the terminal SMC (TSMC) and twisting secondorder SMC are applied for the consensus of second-order MASs with disturbances. In [27], an integral sliding mode approach was used to solve the finite-time consensus problem of second-order MASs with bounded disturbances, however, the chattering problem is ignored.

Motivated by these discussions, we address the second- 
order consensus problem of a class of nonlinear MASs subject to unmeasurable state via the sliding mode control approach. The main contributions of this paper are listed as follows. Firstly, compared with [27], the nonlinear dynamic in MASs is only restricted to be uniformly bounded without needing to know its derivative information, and a finite-time observer and a first-order sliding mode controller are presented for the consensus of secondorder nonlinear MASs with unmeasurable velocity. Secondly, due to the chattering problem in the first-order sliding mode controller, modified consensus controllers based on the boundary layer method and the super-twisting algorithm are presented respectively. In conclusion, this paper investigates the consensus problem of second-order nonlinear MASs under a relaxed assumption of nonlinear dynamic via the finite-time observer and the sliding mode controller.

This paper is organized as follows. In Section 2, the graph theory and the second-order nonlinear MASs are introduced. Distributed observer and controller based on SMC for the consensus of second-order nonlinear multiagent systems are presented in Section 3. Section 4 investigates the modified SMC based on the boundary layer method and the super-twisting algorithm. In Section 5, simulation results are provided and Section 6 concludes this paper.

\section{Problem formulation and preliminaries}

\subsection{Graph theory}

A graph $G=(V, E)$ is used to describe the topology of MASs, $V=\{1,2, \ldots, n\}$ denotes the vertex set and $E \subseteq\{(i, j) \mid i, j \in V, i \neq j\}$ denotes the edge set, respectively. The topology is considered to be directed in this paper. If $(i, j) \in E$, we say the agent $j$ can receive information from its neighbor agent $i$. If node $j \in N_{i}(t)=$ $\{j \mid(j, i) \in E, j \neq i\}$, we say the node $j$ is a neighbour of the agent $i$. If there is a node in graph $G$ which can reach all other nodes, then we say $G$ contains a spanning tree. Define the Laplacian matrix $\boldsymbol{L}(G)$ (simply $\boldsymbol{L}$ ) as $\boldsymbol{L}=\boldsymbol{D}-\boldsymbol{A}$, where $\boldsymbol{A}=\left[a_{i j}\right]$ is the adjacency matrix with $a_{i j}=1$, $\forall(i, j) \in E$ and $a_{i j}=0$ otherwise and $\boldsymbol{D}=\operatorname{diag}\left(\sum_{j \neq i} a_{i j}\right)$ is the degree matrix. Other relevant graph theory knowledge can be found in [28].

Lemma 1 [29] For $x_{i} \in \mathbf{R}(i=1,2, \ldots, n), \alpha \in$ $(0,1]$, then $\left(\sum_{i=1}^{n}\left|x_{i}\right|\right)^{\alpha} \leqslant \sum_{i=1}^{n}\left|x_{i}\right|^{\alpha}$.

\subsection{Problem formulation}

The second-order nonlinear MAS dynamics are described as follows:

$$
\left\{\begin{array}{l}
\dot{x}_{i}(t)=v_{i}(t) \\
\dot{v}_{i}(t)=f_{i}\left(t, x_{i}(t), v_{i}(t)\right)+u_{i}(t)
\end{array}, i=1, \cdots, N\right.
$$

where $x_{i}(t), v_{i}(t) \in \mathbf{R}$ are the position and velocity, respectively. $f_{i}: \mathbf{R} \mapsto \mathbf{R}$ is a continuously differentiable function, $u_{i}(t) \in \mathbf{R}$ is the control input. Although the system is considered to be second-order, the proposed method in this paper can be extended to the high-order case. The following assumption is needed in this paper.

Assumption $1 f_{i}\left(t, x_{i}(t), v_{i}(t)\right)$ is uniformly bounded, i.e., there exists a known $\eta>0$ satisfying

$$
\left|f_{i}\left(t, x_{i}(t), v_{i}(t)\right)\right| \leqslant \eta .
$$

Lemma 2 [30] Consider the following first-order MASs

$$
\dot{x}_{i}=\sum_{j \in N_{i}} a_{i j}\left(x_{j}-x_{i}\right),
$$

then the MASs can achieve consensus, if and only if the system topology contains a spanning tree.

In this paper, the consensus is said to be achieved if and only if the following conditions hold:

$$
\left\{\begin{array}{l}
\lim _{t \rightarrow \infty}\left\|x_{i}-x_{j}\right\|=0 \\
\lim _{t \rightarrow \infty}\left\|v_{i}-v_{j}\right\|=0
\end{array}, \quad i, j \in V .\right.
$$

Remark 1 In this paper, the MASs finally achieve average consensus which means the average value of the initial states, of course it is static consensus because the velocities of agents decay to zero finally.

\section{Main result}

In this section, we first present the second-order sliding mode observer based on the super-twisting algorithm [31] for MASs, and then according to the separation principle [32] and proposed interacted sliding mode surfaces, three distributed sliding mode consensus controllers are designed to eliminate the chatting.

\subsection{Second-order sliding mode observer design}

Since the velocities of agents are assumed to be unmeasurable, we need to estimate the velocity firstly. The supertwisting second-order sliding mode observer as follows by developing the design of the super-twisting sliding mode observer is given as follows:

$$
\left\{\begin{array}{l}
\dot{\hat{x}}_{i}=\widehat{v}_{i}+w_{1} \\
\dot{\hat{v}}_{i}=f_{i}\left(t, \widehat{x}_{i}, \widehat{v}_{i}\right)+u_{i}+w_{2}
\end{array}, \quad i=1, \cdots, N\right.
$$

where $\widehat{x}_{i}, \widehat{v}_{i}$ are the estimation variables of $x_{i}, v_{i}$, and $w_{1}$, $w_{2}$ are auxiliary variables in the following form:

$$
\left\{\begin{array}{l}
w_{1}=\alpha_{1}\left|x_{i}-\widehat{x}_{i}\right|^{1 / 2} \operatorname{sgn}\left(x_{i}-\widehat{x}_{i}\right) \\
w_{2}=\alpha_{2} \operatorname{sgn}\left(x_{i}-\widehat{x}_{i}\right)
\end{array}\right.
$$


where $\alpha_{1}, \alpha_{2}>0$ are to be determined, and $\operatorname{sgn}(\cdot)$ is the following sign function

$$
\operatorname{sgn}\left(\theta_{i}\right)=\left\{\begin{array}{l}
1, \quad \theta_{i}>0 \\
0, \quad \theta_{i}=0 \\
-1, \quad \theta_{i}<0
\end{array} .\right.
$$

Define $\widetilde{x}_{i}=x_{i}-\widehat{x}_{i}, \widetilde{v}_{i}=v_{i}-\widehat{v}_{i}$, and then according to (4) and (5), one can get the error dynamics

$$
\left\{\begin{array}{l}
\dot{\tilde{x}}_{i}=\widetilde{v}_{i}-\alpha_{1}\left|\widetilde{x}_{i}\right|^{1 / 2} \operatorname{sgn}\left(\widetilde{x}_{i}\right) \\
\dot{\widetilde{v}}_{i}=F_{i}\left(t, x_{i}, v_{i}, \widehat{x}_{i}, \widehat{v}_{i}\right)-\alpha_{2} \operatorname{sgn}\left(\widetilde{x}_{i}\right)
\end{array}\right.
$$

where $F_{i}\left(t, x_{i}, v_{i}, \widehat{x}_{i}, \widehat{v}_{i}\right)=f_{i}\left(t, x_{i}, v_{i}\right)-f_{i}\left(t, \widehat{x}_{i}, \widehat{v}_{i}\right)$, and $\left|F_{i}\left(t, x_{i}, v_{i}, \widehat{x}_{i}, \widehat{v}_{i}\right)\right| \leqslant 2 \eta$.

Now we give the following lemma to verify the effectiveness of the proposed observer.

Lemma 3 Suppose that Assumption 1 holds. For the system (1) and the observer (4), if $\alpha_{1}, \alpha_{2}$ satisfy

$$
\alpha_{1}>2, \quad \alpha_{2}>\frac{\alpha_{1}^{3}+4\left(4 \alpha_{1}-8\right) \eta^{2}}{\alpha_{1}\left(4 \alpha_{1}-8\right)}
$$

then the system (6) can converge to the origin in finite time.

Proof Consider the following Lyapunov function

$$
V_{i}\left(\widetilde{x}_{i}, \widetilde{v}_{i}\right)=2 \alpha_{2}\left|\widetilde{x}_{i}\right|+\frac{1}{2}\left(\widetilde{v}_{i}^{\mathrm{T}} \widetilde{v}_{i}+\theta_{i}^{\mathrm{T}} \theta_{i}\right)=\boldsymbol{\xi}_{i}^{\mathrm{T}} \boldsymbol{P} \boldsymbol{\xi}_{i}
$$

where $\theta_{i}^{\mathrm{T}}=-\widetilde{v}_{i}+\alpha_{1}\left|\widetilde{x}_{i}\right|^{1 / 2} \operatorname{sgn}\left(\widetilde{x}_{i}\right), \quad \boldsymbol{\xi}_{i}^{\mathrm{T}}=$ $\left[\left|\widetilde{x}_{i}\right|^{1 / 2} \operatorname{sgn}\left(\widetilde{x}_{i}\right) \quad \widetilde{v}_{i}\right], \boldsymbol{P}=\frac{1}{2}\left[\begin{array}{cc}\alpha_{1}^{2}+4 \alpha_{2} & -\alpha_{1} \\ -\alpha_{1} & 2\end{array}\right]$. Note that $V_{i}\left(\widetilde{x}_{i}, \widetilde{v}_{i}\right)$ is continuous differentiable except $\widetilde{x}_{i}=0$. It is positively definite and radially unbounded if $\alpha_{2}>0$, i.e.

$$
\lambda_{\min }\{\boldsymbol{P}\}\left\|\xi_{i}\right\|^{2} \leqslant V_{i}\left(\widetilde{x}_{i}, \widetilde{v}_{i}\right) \leqslant \lambda_{\max }\{\boldsymbol{P}\}\left\|\xi_{i}\right\|^{2}
$$

where $\lambda_{\min }\{\cdot\}$ and $\lambda_{\max }\{\cdot\}$ are minimum and maximum eigenvalues of the matrix, respectively. $\left\|\xi_{i}\right\|^{2}=\left|\widetilde{x}_{i}\right|+\widetilde{v}_{i}^{2}$ is Euclidean norm of $\xi_{i}$. Furthermore,

$$
\left|\widetilde{x}_{i}\right|^{1 / 2} \leqslant\left\|\xi_{i}\right\| \leqslant \frac{V_{i}\left(\widetilde{x}_{i}, \widetilde{v}_{i}\right)^{1 / 2}}{\lambda_{\min }^{1 / 2}\{\boldsymbol{P}\}}
$$

The derivative of $\boldsymbol{\xi}_{i}$ is

$$
\dot{\boldsymbol{\xi}}_{i}=\left|\widetilde{x}_{i}\right|^{-1 / 2}\left(\boldsymbol{A} \boldsymbol{\xi}_{i}+\boldsymbol{B} \widetilde{F}_{i}\right)
$$

where $\boldsymbol{A}=\left[\begin{array}{cc}-\frac{\alpha_{1}}{2} & \frac{1}{2} \\ -\alpha_{2} & 0\end{array}\right], \boldsymbol{B}=\left[\begin{array}{l}0 \\ 1\end{array}\right]$, and $\widetilde{F}_{i}=$ $\left|\widetilde{x}_{i}\right|^{1 / 2} F\left(t, x_{i}, v_{i}, \widehat{x}_{i}, \widehat{v}_{i}\right)$.

One can get the derivative of $V_{i}\left(\widetilde{x}_{i}, \widetilde{v}_{i}\right)$ along (6) as

$$
\dot{V}_{i}\left(\widetilde{x}_{i}, \widetilde{v}_{i}\right)=\left|\widetilde{x}_{i}\right|^{-1 / 2}\left[\begin{array}{c}
\boldsymbol{\xi}_{i} \\
\widetilde{F}_{i}
\end{array}\right]^{\mathrm{T}} .
$$

$$
\begin{gathered}
{\left[\begin{array}{cc}
\boldsymbol{A}^{\mathrm{T}} \boldsymbol{P}+\boldsymbol{P} \boldsymbol{A} & \boldsymbol{P} \boldsymbol{B} \\
\boldsymbol{B}^{\mathrm{T}} \boldsymbol{P} & \mathbf{0}
\end{array}\right]\left[\begin{array}{c}
\boldsymbol{\xi}_{i} \\
\widetilde{F}_{i}
\end{array}\right] \leqslant} \\
\left|\widetilde{x}_{i}\right|^{-1 / 2}\left(\left[\begin{array}{c}
\boldsymbol{\xi}_{i} \\
\widetilde{F}_{i}
\end{array}\right]^{\mathrm{T}} \cdot\left[\begin{array}{cc}
\boldsymbol{A}^{\mathrm{T}} \boldsymbol{P}+\boldsymbol{P} \boldsymbol{A} & \boldsymbol{P} \boldsymbol{B} \\
\boldsymbol{B}^{\mathrm{T}} \boldsymbol{P} & \mathbf{0}
\end{array}\right] \cdot\left[\begin{array}{c}
\boldsymbol{\xi}_{i} \\
\widetilde{F}_{i}
\end{array}\right]+\right. \\
\left.(2 \eta)^{2}\left|\widetilde{x}_{i}\right|-\widetilde{F}_{i}^{2}\right) \leqslant\left|\widetilde{x}_{i}\right|^{-1 / 2} \boldsymbol{\xi}_{i}^{\mathrm{T}}\left(\boldsymbol{A}^{\mathrm{T}} \boldsymbol{P}+\boldsymbol{P} \boldsymbol{A}+\right. \\
\left.(2 \eta)^{2} \boldsymbol{C}^{\mathrm{T}} \boldsymbol{C}+\boldsymbol{P} \boldsymbol{B} \boldsymbol{B}^{\mathrm{T}} \boldsymbol{P}\right) \boldsymbol{\xi}_{i}=-\left|\widetilde{x}_{i}\right|^{-1 / 2} \boldsymbol{\xi}_{i}^{\mathrm{T}} \boldsymbol{Q} \boldsymbol{\xi}_{i}
\end{gathered}
$$

where $\boldsymbol{C}=\left[\begin{array}{ll}1 & 0\end{array}\right],-\boldsymbol{Q}=\boldsymbol{A}^{\mathrm{T}} \boldsymbol{P}+\boldsymbol{P} \boldsymbol{A}+(2 \eta)^{2} \boldsymbol{C}^{\mathrm{T}} \boldsymbol{C}+$ $\boldsymbol{P} \boldsymbol{B} \boldsymbol{B}^{\mathrm{T}} \boldsymbol{P}$, and

$$
\boldsymbol{Q}=\left[\begin{array}{cc}
\varpi & \frac{\alpha_{1}}{2}-\frac{\alpha_{1}^{2}}{2} \\
\frac{\alpha_{1}}{2}-\frac{\alpha_{1}^{2}}{2} & \frac{\alpha_{1}}{2}-1
\end{array}\right]
$$

where $\varpi=\frac{\alpha_{1}^{3}}{2}-\frac{\alpha_{1}^{2}}{4}+\alpha_{1} \alpha_{2}-4 \eta^{2}$. Define $\alpha_{1}, \alpha_{2}$ as in (7), and then $Q$ is a positive definite matrix. Thus one has

$$
\begin{gathered}
\dot{V}_{i}\left(\widetilde{x}_{i}, \widetilde{v}_{i}\right) \leqslant-\left|\widetilde{x}_{i}\right|^{-1 / 2} \boldsymbol{\xi}_{i}^{\mathrm{T}} \boldsymbol{Q} \boldsymbol{\xi}_{i} \leqslant \\
-\left|\widetilde{x}_{i}\right|^{-1 / 2} \lambda_{\min }\{\boldsymbol{Q}\}\left\|\boldsymbol{\xi}_{i}\right\|^{2} \leqslant-\lambda_{\min }\{\boldsymbol{Q}\}\left\|\boldsymbol{\xi}_{i}\right\| .
\end{gathered}
$$

According to (10) and (13), one has

$$
\dot{V}_{i}\left(\widetilde{x}_{i}, \widetilde{v}_{i}\right) \leqslant-\gamma V_{i}\left(\widetilde{x}_{i}, \widetilde{v}_{i}\right)^{1 / 2}
$$

where $\gamma=\frac{\lambda_{\min }\{\boldsymbol{Q}\}}{\lambda_{\max }\{\boldsymbol{P}\}}$. Following from the comparison principle [33], when $t \geqslant \max _{1 \leqslant i \leqslant N} \frac{2 V_{i}\left(\widetilde{x}_{i}(0), \widetilde{v}_{i}(0)\right)^{1 / 2}}{\gamma}$, we have $V_{i}\left(\widetilde{x}_{i}, \widetilde{v}_{i}\right)=0$. The proof is thus completed.

Remark 2 The finite convergence time is apparently related to the matrix $\boldsymbol{Q}$ and $\boldsymbol{P}$. More directly, it is related to the parameters $\alpha_{1}, \alpha_{2}$ and bound of the nonlinear dynamic $\eta$. In fact, the adaptive sliding mode observer can be further designed to optimize the convergence time according to the adaptive control schemes proposed in [33,34].

\subsection{First-order sliding mode controller design}

In this subsection, the basic idea is to design a sliding mode surface for each agent such that it will converge to the sliding mode surface. Then, if the sliding mode surfaces can converge to one common point, then the consensus is achieved. The first-order sliding mode controller is designed as follows:

$$
u_{i}=\sum_{j \in N_{i}} a_{i j}\left(\widehat{v}_{j}-\widehat{v}_{i}\right)-(\beta+\eta) \operatorname{sgn}\left(s_{i}\right)
$$

where $\beta$ is a positive constant, and the switching function is $s_{i}=\widehat{v}_{i}-k \sum_{j \in N_{i}} a_{i j}\left(\widehat{x}_{j}-\widehat{x}_{i}\right)$, in which $k$ is a positive constant called consensus gain, we make $k=1$ in this paper. 
Then we can obtain the following results.

Theorem 1 Suppose that Assumption 1 holds, and the communication topology contains a spanning tree. Under the first-order sliding mode controller (15), the MAS (1) will achieve consensus asymptotically.

Proof We firstly prove that the system state can reach the sliding mode surface $s_{i}=0$ in finite time. Choosing the Lyapunov function as $V_{i}\left(s_{i}\right)=\frac{1}{2} s_{i}^{\mathrm{T}} s_{i}$ and taking the derivative of $V_{i}$ as

$$
\begin{gathered}
\dot{V}_{i}\left(s_{i}\right)=s_{i} \dot{s}_{i}=s_{i}\left[\dot{\widehat{v}}_{i}-\sum_{j \in N_{i}} a_{i j}\left(\widehat{v}_{j}-\widehat{v}_{i}\right)\right]= \\
s_{i}\left[\dot{v}_{i}-\sum_{j \in N_{i}} a_{i j}\left(v_{j}-v_{i}\right)\right]= \\
s_{i}\left[f_{i}\left(t, x_{i}, v_{i}\right)+u_{i}-\sum_{j \in N_{i}} a_{i j}\left(v_{j}-v_{i}\right)\right]= \\
s_{i}\left[f_{i}\left(t, x_{i}, v_{i}\right)-(\beta+\eta) \operatorname{sgn}\left(s_{i}\right)\right] \leqslant \\
\left|s_{i}\right| \eta-(\beta+\eta)\left|s_{i}\right|=-\beta\left|s_{i}\right|,
\end{gathered}
$$

one can obtain

$$
\dot{V}_{i}(s) \leqslant-\sqrt{2} \beta V_{i}(s)^{1 / 2}<0 .
$$

According to the comparison principle [35], when $t \geqslant$ $\max _{1 \leqslant i \leqslant N} \frac{\sqrt{2 V_{i}\left(s_{i}(0)\right)}}{\gamma}$, one has $s_{i}=v_{i}-\sum_{j \in N_{i}} a_{i j}\left(x_{j}-\right.$ $\left.x_{i}\right)=0$, i.e., $v_{i}(i=1, \cdots, N)$ will converge to their sliding mode surfaces in finite time. Then the MAS (1) can be written as

$$
\dot{x}_{i}=v_{i}=\sum_{j \in N_{i}} a_{i j}\left(x_{j}-x_{i}\right) .
$$

According to Lemma 2, the MAS (1) will achieve consensus, which completes the proof.

Remark 3 According to Lemma 3, the designed second-order sliding mode observer error can converge to the zero within finite time, according to separation principle [32], the design of the observer and controller can be considered and analysed separately, which means the observer states in controller can be replaced by real states in (16).

\subsection{Sliding mode controller based on boundary layer}

In SMC, the chattering problem is one of the important reasons to restrict its application. It is noted that the first-order sliding mode controller proposed in Section 3.2 will generate serious chattering. In order to overcome the chattering problem, Slotine et al. [36] introduced the definition of the boundary layer and the quasi-sliding mode into SMC. The quasi-sliding mode means system trajectory is limited into a $\Delta$ neighbourhood of the ideal sliding mode, where $\Delta$ is called as the boundary layer of the sliding mode.

In this part, we replace the $\operatorname{sgn}\left(s_{i}\right)$ by the saturation function $\operatorname{sat}\left(s_{i}\right)$ to achieve quasi-SMC as follows:

$$
u_{i}=\sum_{j \in N_{i}} a_{i j}\left(\widehat{v}_{j}-\widehat{v}_{i}\right)-(\beta+\eta) \operatorname{sat}\left(s_{i}\right)
$$

where the switching function $s_{i}=v_{i}-\sum_{j \in N_{i}} a_{i j}\left(x_{j}-x_{i}\right)$ with

$$
\operatorname{sat}\left(s_{i}\right)=\left\{\begin{array}{l}
\operatorname{sgn}\left(s_{i}\right), \quad\left|s_{i}\right|>\Delta \\
s_{i} / \Delta, \quad\left|s_{i}\right| \leqslant \Delta
\end{array} .\right.
$$

$\Delta>0$ is the thickness of the boundary layer.

Theorem 2 Suppose that Assumption 1 holds, and the communication topology contains a spanning tree. Under the sliding mode controller based on boundary layer in (18), the MAS (1) will achieve consensus asymptotically.

Proof The proof of the case $\left|s_{i}\right|>\Delta$ is the same as the first-order sliding mode controller design, and we just prove the case $\left|s_{i}\right| \leqslant \Delta$. Choose the Lyapunov function $V_{i}\left(s_{i}\right)=\frac{1}{2} s_{i}^{\mathrm{T}} s_{i}$ and take the derivative of $V_{i}$ as

$$
\begin{gathered}
\dot{V}_{i}\left(s_{i}\right)=s_{i} \dot{s}_{i}= \\
s_{i}\left[f_{i}\left(t, x_{i}, v_{i}\right)-(\beta+\eta) \operatorname{sat}\left(s_{i}\right)\right]= \\
s_{i}\left[f_{i}\left(t, x_{i}, v_{i}\right)-(\beta+\eta) \frac{s_{i}}{\Delta}\right] \leqslant \\
\left|s_{i}\right|\left|f_{i}\left(t, x_{i}, v_{i}\right)\right|-(\beta+\eta) \frac{s_{i}^{2}}{\Delta} \leqslant \\
\Delta \eta-(\beta+\eta) \Delta=-\beta \Delta<0 .
\end{gathered}
$$

As demonstrated in Theorem 1, the MAS (1) will achieve consensus under the controller (18), which completes the proof.

Remark 4 Quasi-SMC can only drive the agent state converge to the neighborhood of sliding surface. Theoretically, the boundary layer thickness $\Delta$ can be designed arbitrarily small to be close to the sliding mode surface, but still cannot guarantee the state converge to the sliding surface. In the worst case, the system state can only reach the boundary of the boundary layer, impacting on the steady state of the system.

\subsection{Second-order sliding mode controller design}

We design the super-twisting second-order sliding mode controller. Compared with the above two controllers, the super-twisting second-order sliding mode controller can keep the invariability of the first-order sliding mode, overcome the chattering problem and improve the system performance. 
The super-twisting second-order sliding mode controller is proposed as follows:

$$
\left\{\begin{array}{l}
u_{i}=\sum_{j \in N_{i}} a_{i j}\left(\widehat{v}_{j}-\widehat{v}_{i}\right)- \\
\beta_{1}\left|s_{i}\right|^{1 / 2} \operatorname{sgn}\left(s_{i}\right)+z_{i} \\
\dot{z}_{i}=-\beta_{2} \operatorname{sgn}\left(s_{i}\right)
\end{array}\right.
$$

where the switching function $s_{i}=v_{i}-\sum_{j \in N_{i}} a_{i j}\left(x_{j}-x_{i}\right)$, and $\beta_{1}, \beta_{2}$ are determined constants.

The derivation of $s_{i}$ is given as follows:

$$
\left\{\begin{array}{l}
\dot{s}_{i}=f_{i}\left(t, x_{i}, v_{i}\right)-\beta_{1}\left|s_{i}\right|^{1 / 2} \operatorname{sgn}\left(s_{i}\right)+z_{i} \\
\dot{z}_{i}=-\beta_{2} \operatorname{sgn}\left(s_{i}\right)
\end{array} .\right.
$$

According to Assumption 1 and $s_{i}=v_{i}-\sum_{j \in N_{i}} a_{i j}\left(x_{j}-\right.$ $\left.x_{i}\right)$, we can rewrite $f_{i}\left(t, x_{i}, v_{i}\right)$ as $f\left(t, s_{i}\right)$. The term $f\left(t, s_{i}\right)$ is uniformly bounded, i.e., there exists a $\varsigma>0$ satisfying $\left|f_{i}\left(t, s_{i}\right)\right| \leqslant \varsigma$. Then (21) can be written as

$$
\left\{\begin{array}{l}
\dot{s}_{i}=f_{i}\left(t, s_{i}\right)-\beta_{1}\left|s_{i}\right|^{1 / 2} \operatorname{sgn}\left(s_{i}\right)+z_{i} \\
\dot{z}_{i}=-\beta_{2} \operatorname{sgn}\left(s_{i}\right)
\end{array} .\right.
$$

According to the controller in (20) and (22), we have the following results.

Theorem 3 Suppose that Assumption 1 holds. For the MAS (1) and the controller (20), if $\beta_{1}$ and $\beta_{2}$ satisfy

$$
\beta_{1}>2, \quad \beta_{2}>\frac{\frac{3}{4} \beta_{1}^{3}-2 \beta_{1}^{2}+\left(\beta_{1}-2\right) \varsigma^{2}}{\left(\beta_{1}+1\right)^{2}-9},
$$

then, the system (22) converges to the origin in finite-time.

Proof Consider the following Lyapunov function

$$
\begin{gathered}
V=\sum V_{i}\left(s_{i}, z_{i}\right)= \\
\sum\left(2 \beta_{2}\left|s_{i}\right|+\frac{1}{2}\left(z_{i}^{\mathrm{T}} z_{i}+v_{i}^{\mathrm{T}} v_{i}\right)\right)=\sum \boldsymbol{\zeta}_{i}^{\mathrm{T}} \boldsymbol{M} \boldsymbol{\zeta}_{i}
\end{gathered}
$$

where $v_{i}^{\mathrm{T}}=\beta_{1}\left|s_{i}\right|^{1 / 2} \operatorname{sgn}\left(s_{i}\right)-z_{i}, \quad \boldsymbol{\zeta}_{i}^{\mathrm{T}}=$ $\left[\left|s_{i}\right|^{1 / 2} \operatorname{sgn}\left(s_{i}\right) \quad z_{i}\right], \boldsymbol{M}=\frac{1}{2}\left[\begin{array}{cc}\beta_{1}^{2}+4 \beta_{2} & -\beta_{1} \\ -\beta_{1} & 2\end{array}\right]$.

The derivative of $\zeta_{i}$ is

$$
\dot{\boldsymbol{\zeta}}_{i}=\left|\widetilde{x}_{i}\right|^{-1 / 2}\left(\widetilde{\boldsymbol{A}} \boldsymbol{\zeta}_{i}+\widetilde{\boldsymbol{B}} \widehat{F}_{i}\right)
$$

where $\widetilde{\boldsymbol{A}}=\left[\begin{array}{cc}-\frac{\beta_{1}}{2} & \frac{1}{2} \\ -\beta_{2} & 0\end{array}\right], \widetilde{\boldsymbol{B}}=\left[\begin{array}{l}1 \\ 0\end{array}\right]$, and $\widehat{F}_{i}=$ $\left|s_{i}\right|^{1 / 2} f\left(t, s_{i}\right)$.

One can get the derivative of $V_{i}\left(s_{i}, z_{i}\right)$ along (6) as

$$
\dot{V}=\sum \dot{V}_{i}\left(s_{i}, z_{i}\right)=\sum\left(\left|s_{i}\right|^{-1 / 2}\left[\begin{array}{c}
\boldsymbol{\zeta}_{i} \\
\widehat{F}_{i}
\end{array}\right]^{\mathrm{T}}\right.
$$

$$
\begin{gathered}
\left.\left[\begin{array}{cc}
\widetilde{\boldsymbol{A}}^{\mathrm{T}} \boldsymbol{M}+\boldsymbol{M} \widetilde{\boldsymbol{A}} & \boldsymbol{M} \widetilde{\boldsymbol{B}} \\
\widetilde{\boldsymbol{B}}^{\mathrm{T}} \boldsymbol{M} & \mathbf{0}
\end{array}\right] \cdot\left[\begin{array}{c}
\boldsymbol{\zeta}_{i} \\
\widehat{F}_{i}
\end{array}\right]\right) \leqslant \\
\sum\left(| s _ { i } | ^ { - 1 / 2 } \left(\left[\begin{array}{c}
\boldsymbol{\zeta}_{i} \\
\widehat{F}_{i}
\end{array}\right]^{\mathrm{T}} .\right.\right.
\end{gathered}
$$

$\left.\left.\left[\begin{array}{cc}\widetilde{\boldsymbol{A}}^{\mathrm{T}} \boldsymbol{M}+\boldsymbol{M} \widetilde{\boldsymbol{A}} & \boldsymbol{M} \widetilde{\boldsymbol{B}} \\ \widetilde{\boldsymbol{B}}^{\mathrm{T}} \boldsymbol{M} & \mathbf{0}\end{array}\right] \cdot\left[\begin{array}{c}\boldsymbol{\zeta}_{i} \\ \widehat{F}_{i}\end{array}\right]+\varsigma^{2}\left|s_{i}\right|-\widehat{F}_{i}^{2}\right)\right) \leqslant$

$\sum\left(\left|s_{i}\right|_{i}^{-1 / 2} \boldsymbol{\zeta}_{i}^{\mathrm{T}}\left(\widetilde{\boldsymbol{A}}^{\mathrm{T}} \boldsymbol{M}+\boldsymbol{M} \widetilde{\boldsymbol{A}}+\right.\right.$

$$
\left.\left.\varsigma^{2} \widetilde{\boldsymbol{C}}^{\mathrm{T}} \widetilde{\boldsymbol{C}}+\boldsymbol{M} \widetilde{\boldsymbol{B}} \widetilde{\boldsymbol{B}}^{\mathrm{T}} \boldsymbol{M}\right) \boldsymbol{\zeta}_{i}\right)=
$$

$$
\sum\left(-\left|s_{i}\right|^{-1 / 2} \boldsymbol{\zeta}_{i}^{\mathrm{T}} \widetilde{\boldsymbol{Q}} \boldsymbol{\zeta}_{i}\right)
$$

where $\widetilde{\boldsymbol{C}}=\left[\begin{array}{ll}1 & 0\end{array}\right],-\widetilde{\boldsymbol{Q}}=\widetilde{\boldsymbol{A}}^{\mathrm{T}} \boldsymbol{M}+\boldsymbol{M} \widetilde{\boldsymbol{A}}+\varsigma^{2} \widetilde{\boldsymbol{C}}{ }^{\mathrm{T}} \widetilde{\boldsymbol{C}}+$ $\boldsymbol{M} \widetilde{\boldsymbol{B}} \widetilde{\boldsymbol{B}}^{\mathrm{T}} \boldsymbol{M}$, and

$$
\widetilde{\boldsymbol{Q}}=\left[\begin{array}{cc}
\pi & \frac{\beta_{1}}{2}-\frac{\beta_{1}^{2}}{2} \\
\frac{\beta_{1}}{2}-\frac{\beta_{1}^{2}}{2} & \frac{\beta_{1}}{2}-1
\end{array}\right]
$$

where $\pi=\frac{\beta_{1}^{3}}{2}-\frac{\beta_{1}^{2}}{4}+\beta_{1} \beta_{2}-\varsigma^{2}+\beta_{1}^{2}+4 \beta_{2}$. Define $\beta_{1}, \beta_{2}$ as in (23), and then $\widetilde{Q}$ is a positive definite matrix. Thus one has

$$
\dot{V}_{i}\left(s_{i}, z_{i}\right) \leqslant-\left|s_{i}\right|^{-1 / 2} \boldsymbol{\zeta}_{i}^{\mathrm{T}} \widetilde{\boldsymbol{Q}} \boldsymbol{\zeta}_{i}<0
$$

According to the similar defination in (9), (13) and Lemma 1, one has

$$
\dot{V} \leqslant \sum \dot{V}_{i}\left(s_{i}, z_{i}\right) \leqslant-\widetilde{\gamma} \sum V_{i}\left(s_{i}, z_{i}\right)^{1 / 2} \leqslant-\widetilde{\gamma} V^{1 / 2}
$$

where $\widetilde{\gamma}=\frac{\lambda_{\min }\{\widetilde{\boldsymbol{Q}}\}}{\lambda_{\max }\{\boldsymbol{M}\}}$. Following from the comparison principle [23], when $t>\max _{1 \leqslant i \leqslant N} \frac{2 V_{i}\left(s_{i}(0), z_{i}(0)\right)^{1 / 2}}{\widetilde{\gamma}}$, we have $V_{i}\left(s_{i}, z_{i}\right)=0$. The proof is thus completed.

Then, we further have the following results.

Theorem 4 Under the Assumption 1 and the topology contains a spanning tree, the super-twisting second-order sliding mode controller (20) guarantees that the MAS (1) can achieve consensus in finite-time.

\section{Simulations}

In this section, some examples are given to show the effectiveness of proposed results. A second-order nonlinear MAS with 10 agents is considered with its communication topology being in Fig. 1. Obviously, there is a directed spanning tree contained in this topology. 


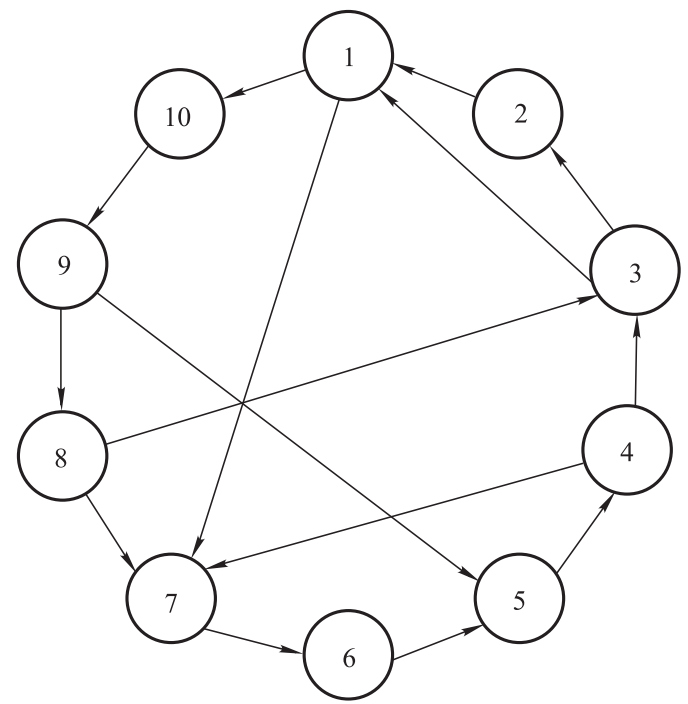

Fig. 1 Topology of MAS

The agent dynamics are described as follows:

$$
\left\{\begin{array}{l}
\dot{x}_{i}(t)=v_{i}(t) \\
\dot{v}_{i}(t)=f_{i}\left(t, x_{i}(t), v_{i}(t)\right)+u_{i}(t)
\end{array}\right.
$$

where $f\left(t, x_{i}(t), v_{i}(t)\right)=\sin \left(x_{i}(t)\right)+\sin \left(v_{i}(t)\right)$, and $\left|f\left(t, x_{i}(t), v_{i}(t)\right)\right| \leqslant 2$, and the Assumption 1 is obviously satisfied. Then we choose $\varsigma=1$. The initial states of the MAS are chosen randomly.

(i) Consensus with second-order sliding mode observer and first-order sliding mode controller

According to Lemma 3, choose $\alpha_{1}=3, \alpha_{2}=8$, and the designed second-order sliding mode observer is given as follows:

$$
\left\{\begin{array}{l}
\dot{\hat{x}}_{i}=\widehat{v}_{i}+3\left|x_{i}-\widehat{x}_{i}\right|^{1 / 2} \operatorname{sgn}\left(x_{i}-\widehat{x}_{i}\right) \\
\dot{\hat{v}}_{i}=f_{i}\left(t, \widehat{x}_{i}, \widehat{v}_{i}\right)+u_{i}+8 \operatorname{sgn}\left(x_{i}-\widehat{x}_{i}\right)
\end{array}\right.
$$

with the designed first-order sliding mode controller as in (15). Let $\beta=1$, then

$$
u_{i}=\sum_{j \in N_{i}} a_{i j}\left(\widehat{v}_{j}-\widehat{v}_{i}\right)-(1+2) \operatorname{sgn}\left(s_{i}\right)
$$

where $s_{i}=\widehat{v}_{i}-\sum_{j \in N_{i}} a_{i j}\left(\widehat{x}_{j}-\widehat{x}_{i}\right)$.

The simulation results are shown in Fig. 2 and Fig. 3. Fig. 2 represents the observation errors of position and velocity of agents. One can find that the second-order sliding mode observer (30) can observe the velocity very well in finite time. For the other two control cases, we omit the similar simulation results. In Fig. 3(a) and Fig. 3(b), one can see under the controller (31), the positions and velocities of the MASs can achieve consensus. In Fig. 3(c), the sliding mode surfaces converge to the same point which lead to the consensus. It is shown in Fig. 3(d) that there exists a serious chattering problem in the control input.

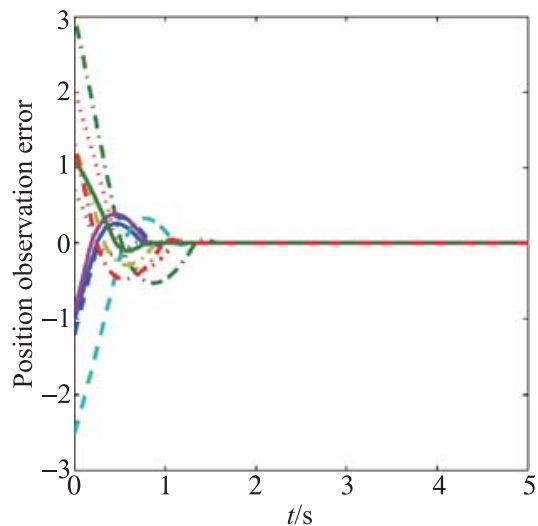

(a) Position observation errors

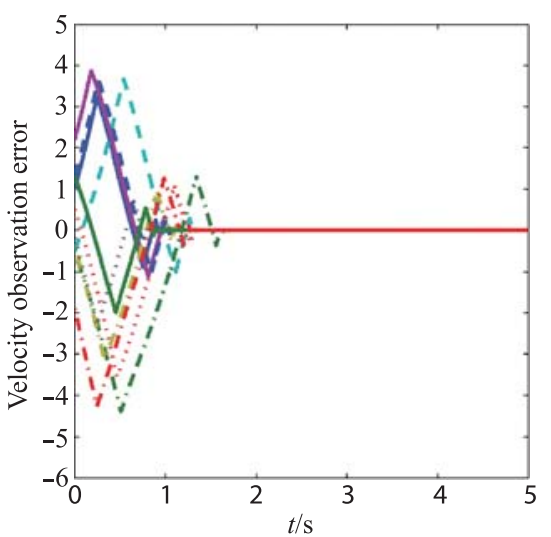

(b) Velocity observation errors

Fig. 2 Observer errors of MASs

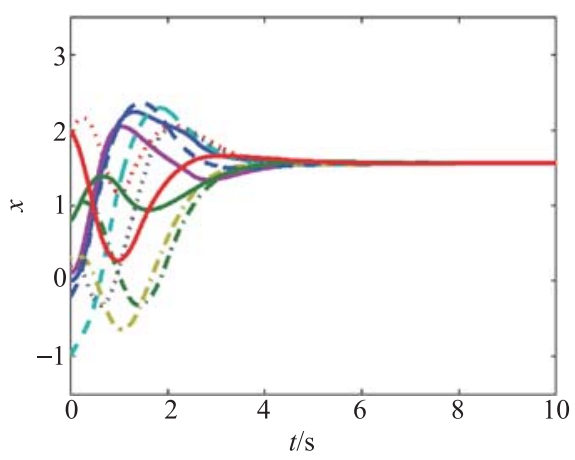

(a) Position

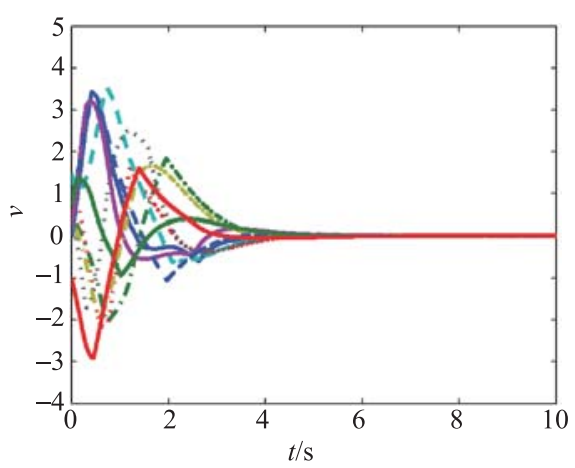

(b) Velocity 


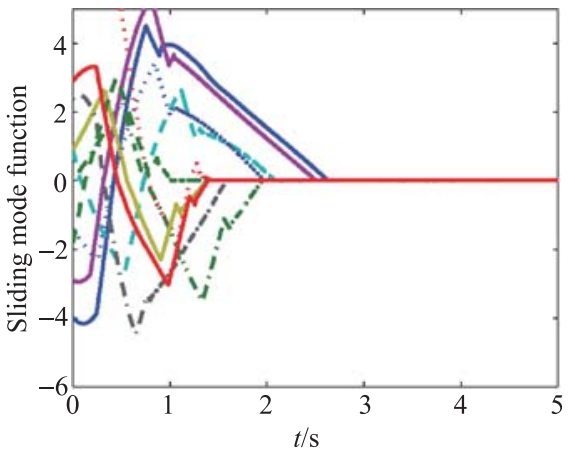

(c) Sliding mode

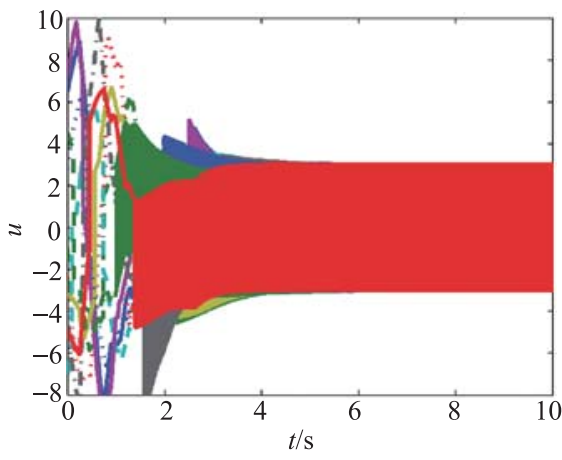

(d) Controller

Fig. 3 Consensus with the first-order SMC in (31)

(ii) Consensus with sliding mode controller based on boundary layer

We consider the sliding mode controller based on the boundary layer as given in (18). Choose the boundary layer thickness as $\Delta=0.01$ and $\beta=1$, then

$$
u_{i}=\sum_{j \in N_{i}} a_{i j}\left(\widehat{v}_{j}-\widehat{v}_{i}\right)-(1+2) \operatorname{sat}\left(s_{i}\right) .
$$

The consensus results are shown in Fig. 4(a) and Fig. 4(b). One can find that the MAS (29) achieve consensus under the controller (32). Moreover, the sliding mode functions also achieve consensus as shown in Fig. 4(c). Fig. 4(d) shows that the sliding mode controller based on the boundary layer can effectively reduce chattering by comparison with the control input of first-order SMC in Fig. 3(d).

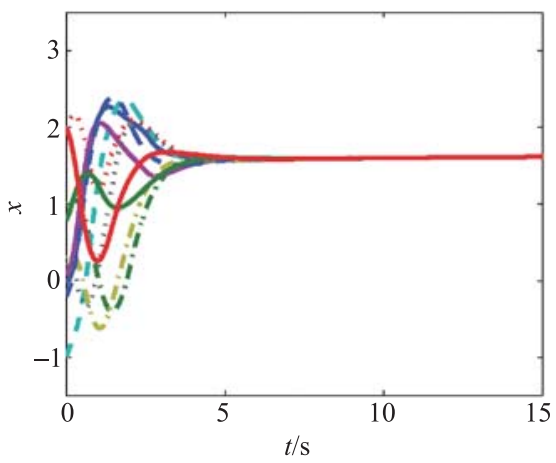

(a) Position

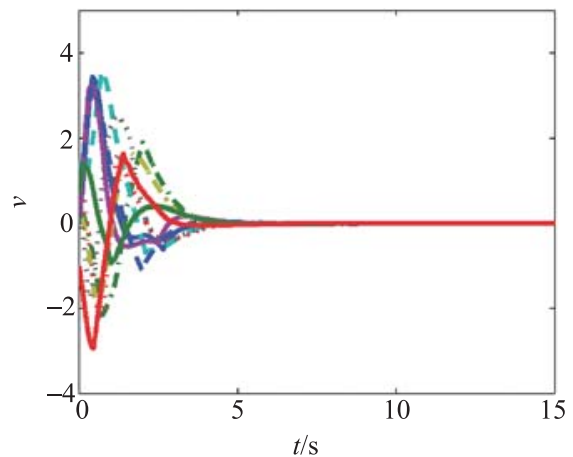

(b) Velocity

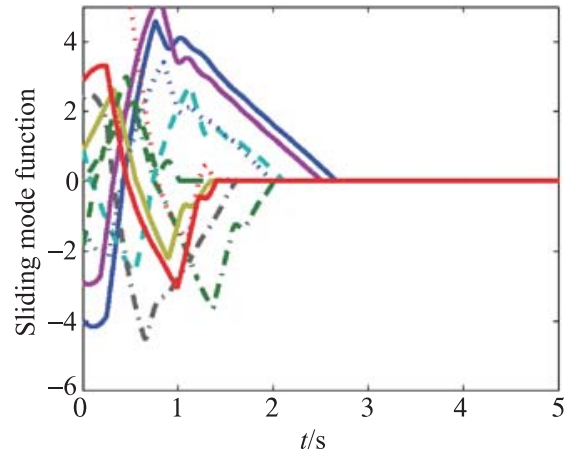

(c) Sliding mode

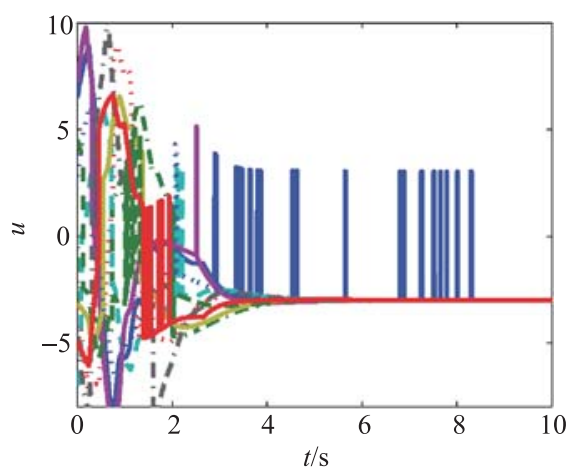

(d) Controller

Fig. 4 Consensus with the boundary layer SMC in (32)

(iii) Consensus with second-order sliding mode controller

In order to balance the accuracy and chattering, we consider the super-twisting second-order sliding mode controller (20), choose the parameters as $\beta_{1}=3, \beta_{2}=9$ according to Theorem 3 , then the controller can be written as follows:

$$
\left\{\begin{array}{l}
u_{i}=\sum_{j \in N_{i}} a_{i j}\left(\widehat{v}_{j}-\widehat{v}_{i}\right)-3\left|s_{i}\right|^{1 / 2} \operatorname{sgn}\left(s_{i}\right)+z_{i} \\
\dot{z}_{i}=-4 \operatorname{sgn}\left(s_{i}\right)
\end{array} .\right.
$$

Obviously, the positions and velocities can achieve the consensus under the controller (30) as shown in Fig. 5(a) and Fig. 5(b). 
In Fig. 5(c), the sliding mode functions convenge to the origin. The control inputs are shown in Fig. 5(d), and one can see that the chatting is greatly reduced by the secondorder SMC. Compared with the previous two controllers, it has much more better control performances both in overcoming the chatting problem and accuracy.

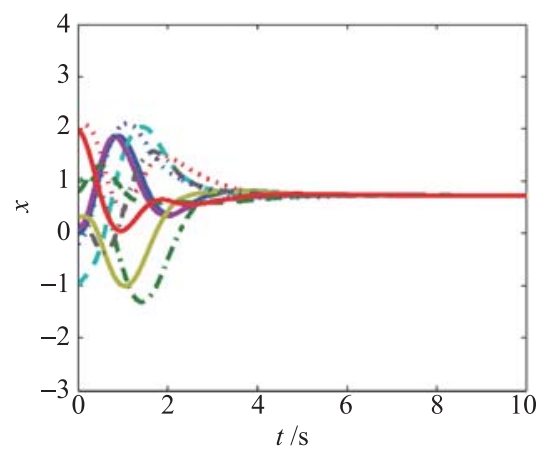

(a) Position

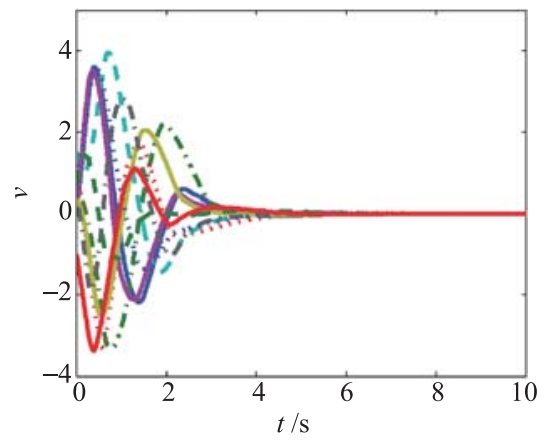

(b) Velocity

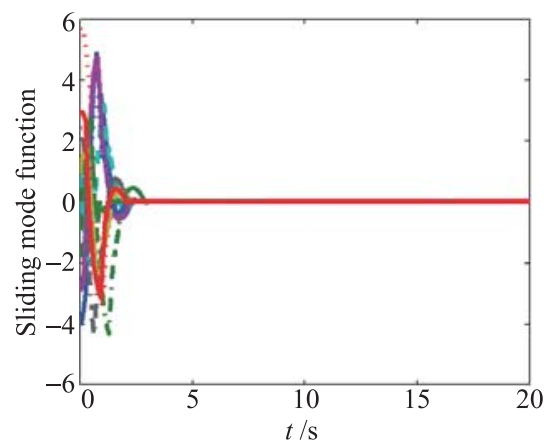

(c) Sliding mode

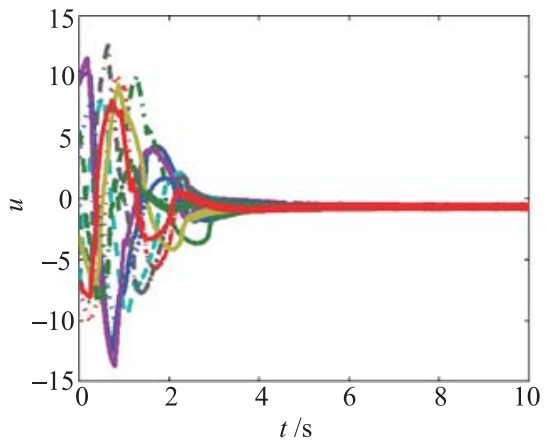

(d) Controller

Fig. 5 Consensus with the second-order SMC in (33)
In order to further explain the advantages of the proposed second-order SMC in this paper, we compare it with the results in [8] where the second-order consensus tracking problem of uncertain multi-vehicle systems without velocity measurements is considered. The results are shown in Fig. 6(a) and Fig. 6(b). Compared with the consensus results as shown in Fig. 5(a) and Fig. 5(b), it is obviously that the convergent speed and robustness under our proposed second-order SMC are better than that of the consensus algorithm in [8].

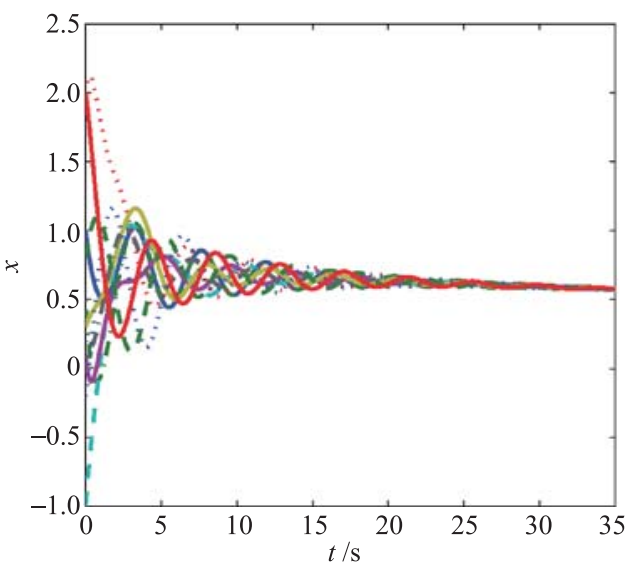

(a) Position

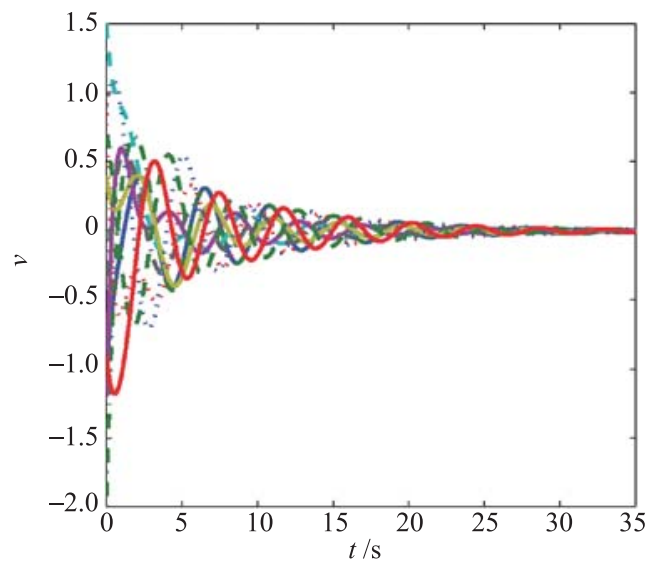

(b) Velocity

Fig. 6 Consensus with the nonlinear robust controller in [8]

\section{Conclusions}

The second-order consensus problem of a class of nonlinear MASs is investigated via sliding mode observer and controller in this paper. Due to the advantages of sliding mode control, discontinuous sliding mode protocols are designed to achieve consensus in spite of the nonlinear dynamics. It is shown that under the designed interacted sliding mode surface and fully distributed protocol based on the local state information of neighboring agents the current agent can be drove to the desired steady state. Finally, 
some simulations illustrate the effectiveness of the proposed schemes.

\section{References}

[1] J. A. Fax, R. M. Murray. Information flow and cooperative control of vehicle formations. IEEE Trans. on Automatic Control, 2004, 49(9): $1465-1476$.

[2] Z. Lin, L. Wang, Z. Han, et al. Distributed formation control of multi-agent systems using complex Laplacian. IEEE Trans. on Automatic Control, 2014, 59(7): 1765 - 1777.

[3] H. Zhang, Z. Chao, Z. Chen. A general alignment repulsion algorithm for flocking of multi-agent systems. IEEE Trans. on Automatic Control, 2011, 56(2): 430-435.

[4] H. S. Su, X. F. Wang, Z. Y. Lin. Flocking of multi-agents with a virtual leader. IEEE Trans. on Automatic Control, 2009, 54(2): $293-307$

[5] X. Luo, X. Li, S. Li, et al. Flocking for multi-agent systems with optimally rigid topology based on information weighted Kalman consensus filter. International Journal of Control, Automation, and Systems, 2016, 15(1): 138-148.

[6] S. Zhao, D. Zelazo. Bearing rigidity and almost global bearingonly formation stabilization. IEEE Trans. on Automatic Control, 2016, 61(5): 1255-1268.

[7] W. Yu, W. Zheng, G. Chen, et al. Second-order consensus in multi-agent dynamical systems with sampled position data. Automatica, 2011, 47(7): 1496-1503.

[8] B. Zhu, Z. Li, H. Liu, et al. Robust second-order tracking of multi-vehicle systems without velocity measurements on directed communication topologies. Proc. of the American Control Conference, 2014: 5414-5419.

[9] P. Lin, Y. Jia. Consensus of second-order discrete-time multiagent systems with nonuniform time-delays and dynamically changing topologies. Automatica, 2009, 45(9): 2154-2158.

[10] Z. Li, Z. Duan, G. Chen, et al. Consensus of multiagent systems and synchronization of complex networks: a unified viewpoint. IEEE Trans. on Systems, Man, and Cybernetics, 2010, 57(1): 213-224.

[11] P. Lin, Y. M. Jia. Robust $\mathrm{H}_{\infty}$ consensus analysis of a class of second-order multi-agent systems with uncertainty. IET Control Theory Applications, 2010, 4(3): 487-498.

[12] Y. Zhang, Y. P. Tian. Consensus of data-sampled multi-agent systems with random communication delay and packet loss. IEEE Trans. on Automatic Control, 2010, 55(4): 939-943.

[13] F. Chen, Z. Chen, L. Xiang, et al. Reaching a consensus via pinning control. Automatica, 2009, 45(5): $1215-1220$.

[14] S. Li, G. Feng, X. Guan, et al. Distributed adaptive pinning control for cooperative linear output regulation of multi-agent systems. Proc. of the 32nd Chinese Control Conference, 2013: $6885-6890$.

[15] J. Bai, G. Wen, A. Rahmani, et al. Distributed consensus tracking for the fractional-order multi-agent systems based on the sliding mode control method. Neurocomputing, 2017, 235(26): $210-216$

[16] L. Zhao, Y. Jia, J. Yu. Adaptive finite-time bipartite consensus for second-order multi-agent systems with antagonistic interactions. Systems \& Control Letters, 2017, 102(4): 22-31.

[17] Z. Li, Z. Duan, F. Lewis. Distributed robust consensus control of multi-agent systems with heterogeneous matching uncertainties. Automatica, 2014, 50(3): 883 -889.

[18] E. Montijano, J. I. Montijano, C. Sagüés, et al. Robust discrete time dynamic average consensus. Automatica, 2014, 50(12): $3131-3138$.

[19] J. Wang, A. Lanzon, I. R. Petersen. Robust cooperative control of multiple heterogeneous negative-imaginary systems. Automatica, 2015, 61(11): 64-72.

[20] J. Gao, L. Feng, Y. Zhang. Improvement of consensus convergence speed for linear multi-agent systems based on state observer. Neurocomputing, 2015, 158(22): 26-31.

[21] J. Hu, J. Cao, J. Yu, et al. Consensus of nonlinear multiagent systems with observer-based protocols. Systems \& Control Letters, 2014, 72(6): $71-79$.

[22] Z. L. Tie. Distributed robust finite-time nonlinear consensus protocols for multi-agent systems. International Journal of Systems Science, 2014, 47(6): 1 - 10.

[23] Y. Zhao, Z. Duan, G. Wen. Distributed finite-time tracking of multiple Euler-Lagrange dynamics without velocity measurements. Proc. of the 32nd Chinese Control Conference, 2013: $6923-6928$

[24] M. Ghasemi, S. Nersesov. Finite-time coordination in multiagent systems using sliding mode control approach. Automatica, 2014, 50(4): $1209-1216$.

[25] S. Khoo, L. Xie, Z. Man. Robust finite-time consensus tracking algorithm for multirobot systems. IEEE/ASME Trans. on Mechatronics, 2009, 14(2): 219-228.

[26] A. Pilloni, A. Pisano, M. Franceschelli, et al. Finite-time consensus for a network of perturbed double integrators by second-order sliding mode technique. Proc. of the IEEE Conference on Decision and Control, 2013: 2145 - 2150.

[27] S. Yu, X. Long. Finite-time consensus for second-order multiagent systems with disturbances by integral sliding mode. Automatica, 2015, 54(4): 158-165.

[28] C. Godsil, G. Royle. Algebraic graph theory. New York: Springer Verlag, 2001

[29] I. Nagesh, C. Edwards. A multivariable super-twisting sliding mode approach. Automatica, 2014, 50(3): $984-988$.

[30] R. Olfati-Saber, R. M. Murray. Consensus problems in networks of agents with switching topology and time-delays. IEEE Trans. on Automic Control, 2004, 49(9): 1520-1533.

[31] A. Levant, L. Fridman. Higher order sliding modes. Sliding mode control in engineering. J. P. Barbot, W. Perruguetti (Eds.). New York: Marcel Dekker, 2002: 53-101.

[32] J. Davila, L. Fridman, A. Levant. Second-order sliding-mode observer for mechanical systems. IEEE Trans. on Automatic Control, 2005, 50(11): $1785-1789$.

[33] Y. Shtessel, M. Taled, F. Plestan. A novel adaptive-gain supertwisting sliding mode controller: methodology and application. Automatica, 2012, 48(5): 759-769.

[34] V. I. Utkin, A. S. Poznyak. Adaptive sliding mode control with application to super-twist algorithm: equivalent control method. Automatica, 2013, 49(1): 39-47.

[35] H. K. Khalil. Nonlinear systems. 3rd. New Jersey: PrenticeHall, 2002.

[36] J. J. Slotine, S. S. Sastry. Tracking control of nonlinear systems using sliding surfaces with application to robot manipulator. International Journal of Control, 1983, 38(2): 465-492.

\section{Biographies}

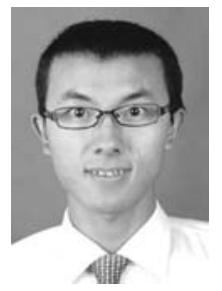

Xiaolei Li was born in 1989. He received his B.E. degree in automation from Yanshan University, Qinhuangdao, China, in 2012, and he is currently pursuing his $\mathrm{Ph} . \mathrm{D}$. degree in control science and engineering at Yanshan University. His current research interests include cooperative control of multi-agent systems and T-S fuzzy control system.

E-mail: 505665918@163.com 


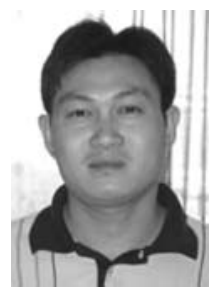

Xiaoyuan Luo was born in 1976. He received his $\mathrm{Ph} . \mathrm{D}$. degree in control theory and control engineering from Yanshan University, Qinhuangdao, China, in 2005. He is currently a professor of the Institute of Electrical Engineering, Yanshan University. His current research interests include cooperative control of multi-agent systems and networked control systems. E-mail: xyluo@ysu.edu.cn

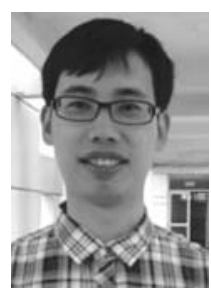

Shaobao Li was born in 1984. He received his B.E degree in automation from Yanshan University, Qinhuangdao, China, in 2006, and Ph.D. degree in mechanical and biomedical engineering from City University of Hong Kong, Hong Kong, in 2014. He is currently a lecturer at Yanshan University. His current research interests include cooperative control of multi-agent systems and topology optimization of wireless sensor networks.

E-mail: shbli84@163.com

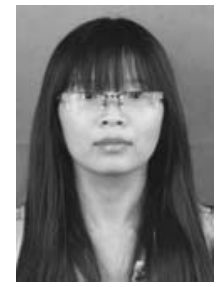

Jianjin Li was born in 1989. She received her B.E. degree in automation from Yanshan University, China, in 2014. She is currently a master student in control theory and control engineering at Yanshan University. Her research interests include cooperative control for multi-agent systems and fault diagnosis of networked system.

E-mail: 875769881@qq.com

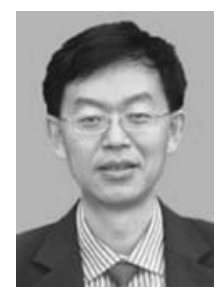

Xinping Guan was born in 1963. He received his M.S. degree in applied mathematics and Ph.D. degree in electrical engineering from Harbin Institute of Technology, Harbin, China, in 1991 and 1999, respectively. He is currently a professor at Shanghai Jiao Tong University, Shanghai, China. His current research interests include networked control systems, wireless sensor networks and applications, and cooperative control of multi-agent systems.

E-mail:xpguan@sjtu.edu.cn 